\title{
Automated Theorem Proving in Projective Geometry with Bracket Algebra
}

\author{
Hongbo Li and Yihong $\mathrm{Wu}$ \\ Institute of Systems Science, Academy of Mathematics and Systems Science \\ Chinese Academy of Sciences, Beijing 100080.
}

\begin{abstract}
We present a method which can produce readable proofs for theorems of constructive type involving points, lines and conics in projective geometry. The method extends Wu's method to bracket algebra and develops the area method of Chou, Gao and Zhang in the framework of projective geometry.
\end{abstract}

\section{Introduction}

Bracket algebra is an important computational tool for projective geometry and has various applications in geometric reasoning, computer vision and robotics. Bracket algebra has been used to prove projective geometric theorems mechanically by several authors in the last decade. Sturmfels and Whitely (1991) proved that the algorithm of straightening laws (Doubilet, Rota and Stein, 1974) is a special algorithm to compute Gröbner bases for polynomials of brackets. So the method of automated theorem proving by Gröbner bases on the coordinate algebra level can be extended to theorem proving by straightening laws on the bracket algebra level. Mourrain and Stolfi (1994) proposed a theorem proving method extending Wu's method from coordinate algebra to bracket algebra. The main feature of their method is delicate applications of the Cramer's rule in linear algebra. Crapo and Richter-Gebert (1994), Richter-Gebert (1995) proposed a method based on bi-quadratic final polynomials. Chou, Gao and Zhang's area method (1994) can also be used to prove theorems in projective geometry, and the areas can be understood as brackets. The method of straightening laws can be applied to all projective geometric theorems, while the latter three methods are applied mainly to incidence theorems. However, proofs produced by the latter three methods are remarkably short, both in the number of steps taken and in the length of polynomials occurred; in one word, the proofs are readable.

Can we get even shorter proofs? Can we extend the methods to theorems involving projective conics? The two problems are the focus of our research on applying bracket algebra to theorem proving. The following are what we have achieved so far: first, we further develop the elimination rules in the area method to produce shorter proofs for incidence theorems; second, we propose a set of elimination rules for theorems involving conics, which turns out to be a method producing specific elimination rules for every specific theorem involving conics; third, we propose a powerful technique called contraction to reduce the size of polynomials of brackets. All these innovations are put together to form a theorem proving method featuring automated production of delicate elimination rules and simplification by contraction. The method extends Wu's method to bracket algebra and develops the area method in the framework of projective geometry. 
We have implemented the method with Maple V Release 4 and have tested over fifty theorems in projective geometry. Our experiments show that the method is capable of producing shorter proofs for incidence theorems, and producing short proofs for theorems involving conics.

\section{Bracket algebra and projective geometry}

Let $\mathcal{V}$ be a vector space of dimension $n$ over a field $\mathcal{F}$. Let $2 \leq d \leq n$. Let $B$ : $\overbrace{\mathcal{V} \times \cdots \times \mathcal{V}}^{d} \longrightarrow \mathcal{F}$ be a mapping which is multilinear, associative and anti-commutative. Then for any $\mathbf{X}_{1}, \ldots, \mathbf{X}_{d} \in \mathcal{V}, B\left(\mathbf{X}_{1}, \ldots, \mathbf{X}_{d}\right)$ is called a bracket of grade $d$ and denoted by $\left[\mathbf{X}_{1} \cdots \mathbf{X}_{d}\right]$.

Let $\mathbf{X}_{1}, \ldots, \mathbf{X}_{m}$ be indeterminates in $\mathcal{V}, m \geq d$. The set of brackets $\left\{\left[\mathbf{X}_{i_{1}} \cdots \mathbf{X}_{i_{d}}\right] \mid 1 \leq\right.$ $\left.i_{j} \leq m\right\}$ generates a polynomial algebra over $\mathcal{F}$ when taken as a set of indeterminates. The quotient of this algebra by the ideal generated by the following Grassmann-Plücker relations is called the bracket algebra of grade $d$ generated by $\mathbf{X}_{1}, \ldots, \mathbf{X}_{m}$ in $\mathcal{V}$ :

$$
\left\{\begin{array}{l|l}
\sum_{k=1}^{d+1}(-1)^{k}\left[\mathbf{X}_{i_{1}} \cdots \mathbf{X}_{i_{d-1}} \mathbf{X}_{j_{k}}\right]\left[\mathbf{X}_{j_{1}} \cdots \mathbf{X}_{j_{k-1}} \mathbf{X}_{j_{k+1}} \cdots \mathbf{X}_{j_{d+1}}\right] & \begin{array}{l}
1 \leq i_{1}<\cdots<i_{d-1} \leq m \\
1 \leq j_{1}<\cdots<j_{d+1} \leq m
\end{array}
\end{array}\right\} .
$$

Bracket algebra is closely related to Grassmann-Cayley algebra. In some sense, bracket algebra is Grassmann-Cayley algebra in scalar form, and Grassmann-Cayley algebra is bracket algebra in multi-vector form. To understand projective geometric computing with bracket algebra one needs some basic knowledge of Grassmann-Cayley algebra. Below is a definition of this algebra. Detailed properties and applications of it in projective geometry can be found in the work of Doubilet, Rota and Stein (1974), Hestenes and Sobczyk (1984), Sturmfels (1993).

Let $\mathcal{V}$ be an $n$-dimensional vector space over the field $\mathcal{F}$. Then $\mathcal{V}$ generates a Grassmann space $\Lambda(\mathcal{V})$ via a multilinear, associative and anti-commutative operator " $\wedge$ " called outer product. The Grassmann space is graded, whose grades range from 0 to $n$. Let $\langle x\rangle_{r}$ denote the $r$-graded part of $x \in \Lambda(\mathcal{V})$. Let $I$ be a fixed element of grade $n$ in $\Lambda(\mathcal{V})$. The following quadratic form

$$
D(x, y)=\frac{\langle x \wedge y\rangle_{n}}{I}, \text { for } x, y \in \Lambda(\mathcal{V})
$$

is non-singular, and induces a linear invertible mapping $i: \Lambda(\mathcal{V}) \longrightarrow \Lambda\left(\mathcal{V}^{*}\right)$, where $\mathcal{V}^{*}$ is the dual vector space of $\mathcal{V}$. Then $\vee=i^{-1} \circ \wedge \circ i$ defines the meet product in $\Lambda(\mathcal{V})$. The Grassmann space $\Lambda(\mathcal{V})$ equipped with the two products " $\wedge$ " and " $\vee$ " is called the Grassmann-Cayley algebra over $\mathcal{V}$.

In this paper we only concern with the real projective plane, so in our bracket algebras, $n=d=3$. The projective plane is the set of one-dimensional subspaces of $\mathcal{R}^{3}$. A projective point can be represented by a nonzero vector in the one-dimensional subspace, and the representation is unique up to a nonzero scale. A projective line is a two-dimensional subspace of $\mathcal{R}^{3}$. It can be represented by the outer product of two vectors that span the two-dimensional subspace uniquely up to a nonzero scale. The line passing through points $\mathbf{A}, \mathbf{B}$ is thus represented by $\mathbf{A} \wedge \mathbf{B}$, and the intersection of lines $\mathbf{A}_{1} \mathbf{B}_{1}, \mathbf{A}_{2} \mathbf{B}_{2}$ is represented 
by $\left(\mathbf{A}_{1} \wedge \mathbf{B}_{1}\right) \vee\left(\mathbf{A}_{2} \wedge \mathbf{B}_{2}\right)$. Let $\mathbf{A}_{1}, \ldots \mathbf{A}_{5}$ be five points determining a conic. Then point $\mathbf{X}$ is on the conic if and only if for some (or equivalently, for any) permutation $i_{1}, \ldots, i_{5}$ of $1, \ldots, 5$,

$$
\frac{\left[\mathbf{X} \mathbf{A}_{i_{1}} \mathbf{A}_{i_{2}}\right]\left[\mathbf{X} \mathbf{A}_{i_{3}} \mathbf{A}_{i_{4}}\right]}{\left[\mathbf{A}_{i_{5}} \mathbf{A}_{i_{1}} \mathbf{A}_{i_{2}}\right]\left[\mathbf{A}_{i_{5}} \mathbf{A}_{i_{3}} \mathbf{A}_{i_{4}}\right]}=\frac{\left[\mathbf{X} \mathbf{A}_{i_{1}} \mathbf{A}_{i_{3}}\right]\left[\mathbf{X} \mathbf{A}_{i_{2}} \mathbf{A}_{i_{4}}\right]}{\left[\mathbf{A}_{i_{5}} \mathbf{A}_{i_{1}} \mathbf{A}_{i_{3}}\right]\left[\mathbf{A}_{i_{5}} \mathbf{A}_{i_{2}} \mathbf{A}_{i_{4}}\right]}
$$

Three points $\mathbf{A}, \mathbf{B}, \mathbf{C}$ are collinear if and only if

$$
[\mathbf{A B C}]=0 .
$$

Three lines $\mathbf{A}_{11} \mathbf{A}_{12}, \mathbf{A}_{21} \mathbf{A}_{22}, \mathbf{A}_{31} \mathbf{A}_{32}$ are concurrent if and only if for any (or equivalently, for some) permutation $i, j, k$ of $1,2,3$,

$$
\left[\mathbf{A}_{i 1} \mathbf{A}_{j 1} \mathbf{A}_{j 2}\right]\left[\mathbf{A}_{i 2} \mathbf{A}_{k 1} \mathbf{A}_{k 2}\right]=\left[\mathbf{A}_{i 2} \mathbf{A}_{j 1} \mathbf{A}_{j 2}\right]\left[\mathbf{A}_{i 1} \mathbf{A}_{k 1} \mathbf{A}_{k 2}\right]
$$

\section{Expansion and contraction in bracket algebra}

There are two transformations in bracket algebra that are used frequently in this paper. They are both derived from the Grassmann-Plücker relations (??). The first tranformation is called expansion. Let $\mathbf{A}_{1}, \mathbf{A}_{2}, \mathbf{A}_{3}$ be three vectors in $\mathcal{R}^{3}$ that are not in the same twodimensional subspace. Let $\mathbf{X}$ be a vector in $\mathcal{R}^{3}$ and $p$ be an expression of brackets involving $\mathbf{X}$. By expansion of $X$ in $p$ with respect to $\left\{\mathbf{A}_{1}, \mathbf{A}_{2}, \mathbf{A}_{3}\right\}$ we mean setting for every bracket $[\mathbf{X Y Z}]$ in $p$

$$
[\mathbf{X Y Z}]=\frac{\left[\mathbf{X A}_{2} \mathbf{A}_{3}\right]\left[\mathbf{Y Z Z} \mathbf{A}_{1}\right]+\left[\mathbf{X} \mathbf{A}_{3} \mathbf{A}_{1}\right]\left[\mathbf{Y Z} \mathbf{A}_{2}\right]+\left[\mathbf{X} \mathbf{A}_{1} \mathbf{A}_{2}\right]\left[\mathbf{Y Z Z} \mathbf{A}_{3}\right]}{\left[\mathbf{A}_{1} \mathbf{A}_{2} \mathbf{A}_{3}\right]} .
$$

The second transformation is called contraction. For any five vectors $\mathbf{A}_{1}, \ldots, \mathbf{A}_{5}$ in $\mathcal{R}^{3}$, we have

$$
\left[\mathbf{A}_{1} \mathbf{A}_{2} \mathbf{A}_{5}\right]\left[\mathbf{A}_{3} \mathbf{A}_{4} \mathbf{A}_{5}\right]+\left[\mathbf{A}_{1} \mathbf{A}_{3} \mathbf{A}_{5}\right]\left[\mathbf{A}_{4} \mathbf{A}_{2} \mathbf{A}_{5}\right]=\left[\mathbf{A}_{1} \mathbf{A}_{4} \mathbf{A}_{5}\right]\left[\mathbf{A}_{3} \mathbf{A}_{2} \mathbf{A}_{5}\right]
$$

Let $p$ be a polynomial of two terms, each term being the product of at least two brackets. If $p$ is reduced to a monomial of brackets by a suitable form of the identity (??), we say $p$ is contracted. The contraction of polynomials of two monomials can be extended to polynomials of any finitely many monomials.

\section{Elimination rules}

Let $\mathbf{X}$ be a point in the projective plane. The following are typical constructions for a point:

P1. $\mathbf{X}$ is a free point.

P2. $\mathbf{X}$ is a semifree point on a line.

P3. $\mathbf{X}$ is the intersection of two lines.

P4. $\mathrm{X}$ is a semifree point on a conic.

P5. $\mathbf{X}$ is at the intersection of a line and a conic.

P6. $\mathbf{X}$ is at the intersection of two conics.

Let $\mathbf{L}$ be a line on the projective plane. The following are typical constructions for a line: 
L1. $\mathbf{L}$ is a free line.

L2. $\mathbf{L}$ is a semifree line passing through a point.

L3. L passes through two points.

L4. $\mathbf{L}$ is a semifree line tangent to a conic.

L5. L passes through a point and is tangent to a conic.

L6. $\mathbf{L}$ is tangent to two conics.

Because of the duality between points and lines in the projective plane, in this paper we only study elimination rules for points. Assume that $p$ is a polynomial of brackets involving point $\mathbf{X}$.

\section{E1. Eliminate a free point.}

Assume that $\mathbf{X}$ is a free point. Let $\mathbf{A}, \mathbf{B}, \mathbf{C}$ be the first three free points other than $\mathbf{X}$ having the largest number of occurrences in $p$. expand $\mathbf{X}$ with respect to $\{\mathbf{A}, \mathbf{B}, \mathbf{C}\}$ in $p$. The nondegenerate condition is $[\mathbf{A B C}] \neq 0$.

\section{E2. Eliminate a semifree point on a line.}

Assume that $\mathbf{X}$ is on line $\mathbf{A B}$. Let $\mathbf{C}$ be the free point outside line $\mathbf{A B}$ that has the largest number of occurrences in $p$. expand $\mathbf{X}$ with respect to $\{\mathbf{A}, \mathbf{B}, \mathbf{C}\}$ in $p$. The nondegenerate condition is $[\mathbf{A B C}] \neq 0$.

\section{E3. Eliminate the intersection of two lines.}

Assume that $\mathbf{X}$ is the intersection of lines $\mathbf{A}_{11} \mathbf{A}_{12}, \mathbf{A}_{21} \mathbf{A}_{22}$. For every bracket $\left[\mathbf{X} \mathbf{A}_{31} \mathbf{A}_{32}\right]$ in $p$, do the following: if $\left[\mathbf{A}_{11} \mathbf{A}_{31} \mathbf{A}_{32}\right]\left[\mathbf{A}_{12} \mathbf{A}_{31} \mathbf{A}_{32}\right]=0$, set

$$
\left[\mathbf{X A}_{31} \mathbf{A}_{32}\right]=\left[\mathbf{A}_{11} \mathbf{A}_{21} \mathbf{A}_{22}\right]\left[\mathbf{A}_{12} \mathbf{A}_{31} \mathbf{A}_{32}\right]-\left[\mathbf{A}_{12} \mathbf{A}_{21} \mathbf{A}_{22}\right]\left[\mathbf{A}_{11} \mathbf{A}_{31} \mathbf{A}_{32}\right]
$$

else if $\left[\mathbf{A}_{21} \mathbf{A}_{31} \mathbf{A}_{32}\right]\left[\mathbf{A}_{22} \mathbf{A}_{31} \mathbf{A}_{32}\right]=0$, set

$$
\left[\mathbf{X A}_{31} \mathbf{A}_{32}\right]=-\left[\mathbf{A}_{21} \mathbf{A}_{11} \mathbf{A}_{12}\right]\left[\mathbf{A}_{12} \mathbf{A}_{31} \mathbf{A}_{32}\right]+\left[\mathbf{A}_{22} \mathbf{A}_{11} \mathbf{A}_{12}\right]\left[\mathbf{A}_{11} \mathbf{A}_{31} \mathbf{A}_{32}\right]
$$

else if $\left[\mathbf{A}_{11} \mathbf{A}_{12} \mathbf{A}_{31}\right]\left[\mathbf{A}_{11} \mathbf{A}_{12} \mathbf{A}_{32}\right]\left[\mathbf{A}_{21} \mathbf{A}_{22} \mathbf{A}_{31}\right]\left[\mathbf{A}_{21} \mathbf{A}_{22} \mathbf{A}_{32}\right]=0$, set

$$
\left[\mathbf{X A}_{31} \mathbf{A}_{32}\right]=\left[\mathbf{A}_{11} \mathbf{A}_{12} \mathbf{A}_{31}\right]\left[\mathbf{A}_{21} \mathbf{A}_{22} \mathbf{A}_{32}\right]-\left[\mathbf{A}_{11} \mathbf{A}_{12} \mathbf{A}_{32}\right]\left[\mathbf{A}_{21} \mathbf{A}_{22} \mathbf{A}_{31}\right] ;
$$

else, let \# $\mathbf{A}$ be the number of lines passing through point $\mathbf{A}$ in the geometric configuration, then if $\# \mathbf{A}_{i 1}+\# \mathbf{A}_{i 2}=\max \left(\# \mathbf{A}_{j 1}+\# \mathbf{A}_{j 2} \mid 1 \leq j \leq 3\right)$, let $i, j, k$ be an even permutation of $1,2,3$ and set

$$
\left[\mathbf{X} \mathbf{A}_{31} \mathbf{A}_{32}\right]=\left[\mathbf{A}_{i 1} \mathbf{A}_{j 1} \mathbf{A}_{j 2}\right]\left[\mathbf{A}_{i 2} \mathbf{A}_{k 1} \mathbf{A}_{k 2}\right]-\left[\mathbf{A}_{i 2} \mathbf{A}_{j 1} \mathbf{A}_{j 2}\right]\left[\mathbf{A}_{i 1} \mathbf{A}_{k 1} \mathbf{A}_{k 2}\right]
$$

There is no nondegenerate condition.

\section{E4. Eliminate a semifree point on a conic.}

Assume that $\mathbf{X}$ is on conic $\mathbf{A}_{1} \mathbf{A}_{2} \mathbf{A}_{3} \mathbf{A}_{4} \mathbf{A}_{5}$. There are two steps in establishing an elimination rule for $\mathbf{X}$. The first step is to set up a suitable equation $e=0$ for $\mathbf{X}$. Ordering the A's as follows: $\mathbf{A}_{i} \prec \mathbf{A}_{j}$ if $\mathbf{A}_{i}$ precedes $\mathbf{A}_{j}$ in the list of construction types 
P1-P6, or $\mathbf{A}_{i}, \mathbf{A}_{j}$ are of the same construction type but $\mathbf{A}_{i}$ occurs more than $\mathbf{A}_{j}$ in $p$. Let $\mathbf{A}_{i_{1}} \prec \mathbf{A}_{i_{2}} \prec \mathbf{A}_{i_{3}} \prec \mathbf{A}_{i_{4}} \prec \mathbf{A}_{i_{5}}$ for some permutation $i_{1}, \ldots, i_{5}$ of $1, \ldots, 5$. Then

$$
\begin{aligned}
e= & {\left[\mathbf{A}_{i_{1}} \mathbf{A}_{i_{2}} \mathbf{A}_{i_{4}}\right]\left[\mathbf{A}_{i_{1}} \mathbf{A}_{i_{2}} \mathbf{A}_{i_{5}}\right]\left[\mathbf{A}_{i_{3}} \mathbf{A}_{i_{4}} \mathbf{A}_{i_{5}}\right]\left[\mathbf{X} \mathbf{A}_{i_{1}} \mathbf{A}_{i_{3}}\right]\left[\mathbf{X} \mathbf{A}_{i_{2}} \mathbf{A}_{i_{3}}\right] } \\
- & {\left[\mathbf{A}_{i_{1}} \mathbf{A}_{i_{3}} \mathbf{A}_{i_{4}}\right]\left[\mathbf{A}_{i_{1}} \mathbf{A}_{i_{3}} \mathbf{A}_{i_{5}}\right]\left[\mathbf{A}_{i_{2}} \mathbf{A}_{i_{4}} \mathbf{A}_{i_{5}}\right]\left[\mathbf{X} \mathbf{A}_{i_{1}} \mathbf{A}_{i_{2}}\right]\left[\mathbf{X} \mathbf{A}_{i_{2}} \mathbf{A}_{i_{3}}\right] } \\
& +\left[\mathbf{A}_{i_{1}} \mathbf{A}_{i_{4}} \mathbf{A}_{i_{5}}\right]\left[\mathbf{A}_{i_{2}} \mathbf{A}_{i_{3}} \mathbf{A}_{i_{4}}\right]\left[\mathbf{A}_{i_{2}} \mathbf{A}_{i_{3}} \mathbf{A}_{i_{5}}\right]\left[\mathbf{X} \mathbf{A}_{i_{1}} \mathbf{A}_{i_{2}}\right]\left[\mathbf{X} \mathbf{A}_{i_{1}} \mathbf{A}_{i_{3}}\right] .
\end{aligned}
$$

The second step occurs when $\left\{\mathbf{A}_{i_{4}}, \mathbf{A}_{i_{5}}\right\}$ contains intersections of lines or semifree points on lines. Such points are eliminated from $e$ by rules $\mathbf{E 3}$ and $\mathbf{E 2}$, and then $e$ is contracted and bracket factors not involving $\mathbf{X}$ are removed. The result is still denoted by $e$.

To eliminate $\mathbf{X}$ from $p$, there are two steps. The first step is to expand $\mathbf{X}$ with respect to $\left\{\mathbf{A}_{i_{1}}, \mathbf{A}_{i_{2}}, \mathbf{A}_{i_{3}}\right\}$ in $p$ and obtain a polynomial $p^{\prime}$ from $p$ after removing the common denominator. The second step is to make pseudo-division to $p^{\prime}$ by $e$, taking one of $\left[\mathbf{X} \mathbf{A}_{i_{1}} \mathbf{A}_{i_{2}}\right]$, $\left[\mathbf{X} \mathbf{A}_{i_{1}} \mathbf{A}_{i_{3}}\right],\left[\mathbf{X} \mathbf{A}_{i_{2}} \mathbf{A}_{i_{3}}\right]$ as the leading variable.

The nondegenerate condition is composed of $\left[\mathbf{A}_{i_{1}} \mathbf{A}_{i_{2}} \mathbf{A}_{i_{3}}\right] \neq 0$, the factors removed during establishing the elimination rule being nonzero, and the leading coefficients for the pseudodivision being nonzero.

\section{E5. Eliminate a point at the intersection of a line and a conic.}

Assume that points $\mathbf{X}, \mathbf{A}$ are at the intersection of line $\mathbf{A B}$ and conic $\mathbf{A} \mathbf{A}_{1} \mathbf{A}_{2} \mathbf{A}_{3} \mathbf{A}_{4}$. There are two steps in establishing an elimination rule for $\mathbf{X}$. The first step is to set up two suitable equations $e_{1}=0, e_{2}=0$ for $\mathbf{X}$. Ordering $\mathbf{A}_{1}, \mathbf{A}_{2}, \mathbf{A}_{3}, \mathbf{A}_{4}$ as follows: $\mathbf{A}_{i} \prec \mathbf{A}_{j}$ if $\mathbf{A}_{i}$ precedes $\mathbf{A}_{j}$ in the list of construction types $\mathbf{P 1 -} \mathbf{P 6}$, or $\mathbf{A}_{i}, \mathbf{A}_{j}$ are of the same construction type but $\mathbf{A}_{i}$ occurs more than $\mathbf{A}_{j}$ in $p$. Let $\mathbf{A}_{i_{1}} \prec \mathbf{A}_{i_{2}} \prec \mathbf{A}_{i_{3}} \prec \mathbf{A}_{i_{4}}$ for some permutation $i_{1}, \ldots, i_{4}$ of $1, \ldots, 4$. Then

$$
\begin{aligned}
& e_{1}=\left[\mathbf{A A}_{i_{1}} \mathbf{B}\right]\left[\mathbf{X} \mathbf{A} \mathbf{A}_{i_{2}}\right]-\left[\mathbf{A} \mathbf{A}_{i_{2}} \mathbf{B}\right]\left[\mathbf{X} \mathbf{A} \mathbf{A}_{i_{1}}\right], \\
& e_{2}=\left[\mathbf{A} \mathbf{A}_{i_{1}} \mathbf{A}_{i_{3}}\right]\left[\mathbf{A} \mathbf{A}_{i_{1}} \mathbf{A}_{i_{4}}\right]\left[\mathbf{A}_{i_{2}} \mathbf{A}_{i_{3}} \mathbf{A}_{i_{4}}\right]\left[\mathbf{A} \mathbf{A}_{i_{2}} \mathbf{B}\right]\left[\mathbf{X} \mathbf{A}_{i_{1}} \mathbf{A}_{i_{2}}\right] \\
& -\left[\mathbf{A} \mathbf{A}_{i_{2}} \mathbf{A}_{i_{3}}\right]\left[\mathbf{A} \mathbf{A}_{i_{2}} \mathbf{A}_{i_{4}}\right]\left[\mathbf{A}_{i_{1}} \mathbf{A}_{i_{3}} \mathbf{A}_{i_{4}}\right]\left[\mathbf{A} \mathbf{A}_{i_{1}} \mathbf{B}\right]\left[\mathbf{X} \mathbf{A}_{i_{1}} \mathbf{A}_{i_{2}}\right]
\end{aligned}
$$

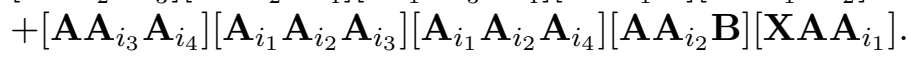

The second step occurs when $\left\{\mathbf{A}_{i_{3}}, \mathbf{A}_{i_{4}}, \mathbf{B}\right\}$ contains intersections of lines or semifree points on lines. Such points are eliminated from $e_{1}, e_{2}$ by rules $\mathbf{E 3}$ and $\mathbf{E 2}$, and then $e_{1}, e_{2}$ are contracted and bracket factors not involving $\mathbf{X}$ are removed. The result is in the form

$$
\begin{aligned}
& e_{1}=\lambda_{1}\left[\mathbf{X} \mathbf{A}_{i_{2}}\right]-\lambda_{2}\left[\mathbf{X A A} \mathbf{A}_{i_{1}}\right] \\
& e_{2}=\lambda_{3}\left[\mathbf{X A}_{i_{1}} \mathbf{A}_{i_{2}}\right]-\lambda_{4}\left[\mathbf{X} \mathbf{A} \mathbf{A}_{i_{1}}\right]
\end{aligned}
$$

where the $\lambda$ 's are polynomials of brackets not involving $\mathbf{X}$.

To eliminate $\mathbf{X}$ from $p$, there are two steps. The first step is to expand $\mathbf{X}$ with respect to $\left\{\mathbf{A}, \mathbf{A}_{i_{1}}, \mathbf{A}_{i_{2}}\right\}$ in $p$ and obtain a polynomial $p^{\prime}$ from $p$ after removing the common denominator. The second step is to set

$$
\left[\mathbf{X A A}_{i_{2}}\right]=\lambda_{2} \lambda_{3}, \quad\left[\mathbf{X} \mathbf{A}_{i_{1}} \mathbf{A}_{i_{2}}\right]=\lambda_{1} \lambda_{4}, \quad\left[\mathbf{X A} \mathbf{A}_{i_{1}}\right]=\lambda_{1} \lambda_{3}
$$

and obtain a polynomial $p^{\prime \prime}$ from $p^{\prime}$. 
The nondegenerate condition is composed of $\left[\mathbf{A} \mathbf{A}_{i_{1}} \mathbf{A}_{i_{2}}\right] \neq 0$, the factors removed during establishing the elimination rule being nonzero, and the leading coefficients for the pseudodivision being nonzero.

\section{E6. Eliminate a point at the intersection of two conics.}

Assume that points $\mathbf{X}, \mathbf{A}_{1}, \mathbf{A}_{2}, \mathbf{A}_{3}$ are at the intersection of conics $\mathbf{A}_{1} \mathbf{A}_{2} \mathbf{A}_{3} \mathbf{B}_{1} \mathbf{B}_{2}$ and $\mathbf{A}_{1} \mathbf{A}_{2} \mathbf{A}_{3} \mathbf{C}_{1} \mathbf{C}_{2}$. There are two steps in establishing an elimination rule for $\mathbf{X}$. The first step is to set up two equations $e_{1}=0, e_{2}=0$ for $\mathbf{X}$ :

$$
\begin{aligned}
& e_{1}=\lambda_{2}\left[\mathbf{X} \mathbf{A}_{1} \mathbf{A}_{3}\right]-\lambda_{1}\left[\mathbf{X} \mathbf{A}_{1} \mathbf{A}_{2}\right] \\
& e_{2}=\lambda_{3}\left[\mathbf{X} \mathbf{A}_{2} \mathbf{A}_{3}\right]-\lambda_{1}\left[\mathbf{X} \mathbf{A}_{1} \mathbf{A}_{2}\right]
\end{aligned}
$$

where

$$
\begin{array}{cc}
\lambda_{1}= & {\left[\mathbf{A}_{1} \mathbf{A}_{3} \mathbf{B}_{1}\right]\left[\mathbf{A}_{1} \mathbf{A}_{3} \mathbf{B}_{2}\right]\left[\mathbf{A}_{2} \mathbf{B}_{1} \mathbf{B}_{2}\right]\left[\mathbf{A}_{1} \mathbf{C}_{1} \mathbf{C}_{2}\right]\left[\mathbf{A}_{2} \mathbf{A}_{3} \mathbf{C}_{1}\right]\left[\mathbf{A}_{2} \mathbf{A}_{3} \mathbf{C}_{2}\right]} \\
& -\left[\mathbf{A}_{1} \mathbf{B}_{1} \mathbf{B}_{2}\right]\left[\mathbf{A}_{2} \mathbf{A}_{3} \mathbf{B}_{1}\right]\left[\mathbf{A}_{2} \mathbf{A}_{3} \mathbf{B}_{2}\right]\left[\mathbf{A}_{1} \mathbf{A}_{3} \mathbf{C}_{1}\right]\left[\mathbf{A}_{1} \mathbf{A}_{3} \mathbf{C}_{2}\right]\left[\mathbf{A}_{2} \mathbf{C}_{1} \mathbf{C}_{2}\right], \\
\lambda_{2}= & {\left[\mathbf{A}_{1} \mathbf{C}_{1} \mathbf{C}_{2}\right]\left[\mathbf{A}_{2} \mathbf{A}_{3} \mathbf{C}_{1}\right]\left[\mathbf{A}_{2} \mathbf{A}_{3} \mathbf{C}_{2}\right]\left[\mathbf{A}_{1} \mathbf{A}_{2} \mathbf{B}_{1}\right]\left[\mathbf{A}_{1} \mathbf{A}_{2} \mathbf{B}_{2}\right]\left[\mathbf{A}_{3} \mathbf{B}_{1} \mathbf{B}_{2}\right]} \\
& -\left[\mathbf{A}_{1} \mathbf{A}_{2} \mathbf{C}_{1}\right]\left[\mathbf{A}_{1} \mathbf{A}_{2} \mathbf{C}_{2}\right]\left[\mathbf{A}_{3} \mathbf{C}_{1} \mathbf{C}_{2}\right]\left[\mathbf{A}_{1} \mathbf{B}_{1} \mathbf{B}_{2}\right]\left[\mathbf{A}_{2} \mathbf{A}_{3} \mathbf{B}_{1}\right]\left[\mathbf{A}_{2} \mathbf{A}_{3} \mathbf{B}_{2}\right], \\
\lambda_{3}= & {\left[\mathbf{A}_{1} \mathbf{A}_{3} \mathbf{C}_{1}\right]\left[\mathbf{A}_{1} \mathbf{A}_{3} \mathbf{C}_{2}\right]\left[\mathbf{A}_{2} \mathbf{C}_{1} \mathbf{C}_{2}\right]\left[\mathbf{A}_{1} \mathbf{A}_{2} \mathbf{B}_{1}\right]\left[\mathbf{A}_{1} \mathbf{A}_{2} \mathbf{B}_{2}\right]\left[\mathbf{A}_{3} \mathbf{B}_{1} \mathbf{B}_{2}\right]} \\
& -\left[\mathbf{A}_{1} \mathbf{A}_{2} \mathbf{C}_{1}\right]\left[\mathbf{A}_{1} \mathbf{A}_{2} \mathbf{C}_{2}\right]\left[\mathbf{A}_{3} \mathbf{C}_{1} \mathbf{C}_{2}\right]\left[\mathbf{A}_{1} \mathbf{A}_{3} \mathbf{B}_{1}\right]\left[\mathbf{A}_{1} \mathbf{A}_{3} \mathbf{B}_{2}\right]\left[\mathbf{A}_{2} \mathbf{B}_{1} \mathbf{B}_{2}\right] .
\end{array}
$$

The second step occurs when $\left\{\mathbf{B}_{1}, \mathbf{B}_{2}, \mathbf{C}_{1}, \mathbf{C}_{2}\right\}$ contains intersections of lines or semifree points on lines. Such points are eliminated from $e_{1}, e_{2}$ by rules $\mathbf{E} 3$ and $\mathbf{E 2}$, and then $e_{1}, e_{2}$ are contracted and bracket factors not involving $\mathbf{X}$ are removed. The result is in same the form as (??).

To eliminate $\mathbf{X}$ from $p$, there are two steps. The first step is to expand $\mathbf{X}$ with respect to $\left\{\mathbf{A}_{1}, \mathbf{A}_{2}, \mathbf{A}_{3}\right\}$ in $p$ and obtain a polynomial $p^{\prime}$ from $p$ after removing the common denominator. The second step is to set

$$
\left[\mathbf{X A}_{1} \mathbf{A}_{3}\right]=\lambda_{1} \lambda_{3}, \quad\left[\mathbf{X A}_{2} \mathbf{A}_{3}\right]=\lambda_{1} \lambda_{2}, \quad\left[\mathbf{X} \mathbf{A}_{1} \mathbf{A}_{2}\right]=\lambda_{2} \lambda_{3}
$$

and obtain a polynomial $p^{\prime \prime}$ from $p^{\prime}$.

The nondegenerate condition is composed of $\left[\mathbf{A}_{1} \mathbf{A}_{2} \mathbf{A}_{3}\right] \neq 0$, the factors removed during establishing the elimination rule being nonzero, and the leading coefficients for the pseudodivision being nonzero.

Among the six elimination rules, E1-E3 further develops the corresponding elimination rules in the area method, with closer attention paid to the geometric configuration related

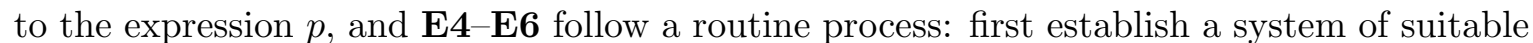
equations depending on $p$, then simplify the equations by the elimination rules E3 and E2.

\section{A theorem proving algorithm}

In this section we present a theorem proving algorithm based on the elimination rules E1-E6. The algorithm is complete for the class of theorems in two-dimensional projective geometry whose constructions of points belong to P1-P6. 
Input. An order of points for both construction and elimination, and a list of constructions of the type P1-P6. Free points are required to precede other points. A conclusion that can be expressed as $c o n c=0$, where conc is a polynomial of brackets.

Step 1. [Preprocess] Change every collinearity constraint in the constructions into a computing rule for brackets by (??). Change the conclusion into the polynomial conc.

Step 2. [Eliminate non-free points] Let ord be the sequence of non-free points. For $\mathbf{X}$ from the last element to the first in ord, do the following sequentially:

(a) If $\mathbf{X}$ is a semifree point on a line, contract conc and denote the result by conc as well. If it equals zero, go to the Output.

(b) Eliminate $\mathbf{X}$ in conc according to its construction type. The result is still denoted by conc. If it equals zero, go to the Output.

(c) Remove monomial factors from conc when there are also polynomial factors in conc.

Step 3. [Eliminate free points] contract conc and denote the result by conc as well. If it equals zero, go to the Output; otherwise let $\mathbf{A}, \mathbf{B}, \mathbf{C}$ be the first three free points having the largest number of occurrences in conc, then expand all free points other than $\mathbf{A}, \mathbf{B}, \mathbf{C}$ in conc with respect to $\{\mathbf{A}, \mathbf{B}, \mathbf{C}\}$. The result must be zero because what is being proved is a theorem.

Output. Print the proving procedure, including the original conc, every elimination and the corresponding elimination rules, every successful contraction, every expansion and every removal of monomial factors. Provide the nondegenerate condition, which is a set of inequalities.

The completeness of the algorithm is guaranteed by Step 3, where essentially a global coordinate system of $\mathcal{R}^{3}$ is used. Step $\mathbf{2}$ (c) is not essential for the algorithm, but can contribute to simplifying later process. In our experiments, it never occurs that the conclusion polynomial is reduced to zero because some monomial factor turns out to be zero when there also exist polynomial factors at the same time.

We have implemented the algorithm with Maple V Release 4 and have tested over fifty theorems in projective geometry. It appears that generally a proof can finish before free points are eliminated, and most strikingly, sometimes even before semifree points on lines are eliminated. Below we give two examples. The first is an incidence theorem and the second is a theorem on conics. Two proofs are produced for the first theorem, both of which finish before a semifree point on a line is eliminated. Four proofs can be produced for the second theorem, all of which finish before free points are eliminated. In the proving process, factors that are to be removed are underlined, and the leftover after each removal appears on the right-hand side of the symbol " $\simeq$ ".

Example 1. [Desargues Theorem] 1, 2, 3, 4, $\mathbf{5}$ are five free points in the projective plane. Point 6 is on line 13, and points 7, 8, 9, $\mathbf{0}$ are intersections of line pairs 45 and $\mathbf{1 2}, \mathbf{1 5}$ and $\mathbf{2 4}, 38$ and 56, 49 and 23 , respectively. Then $6,7,0$ are collinear. 
Proof:

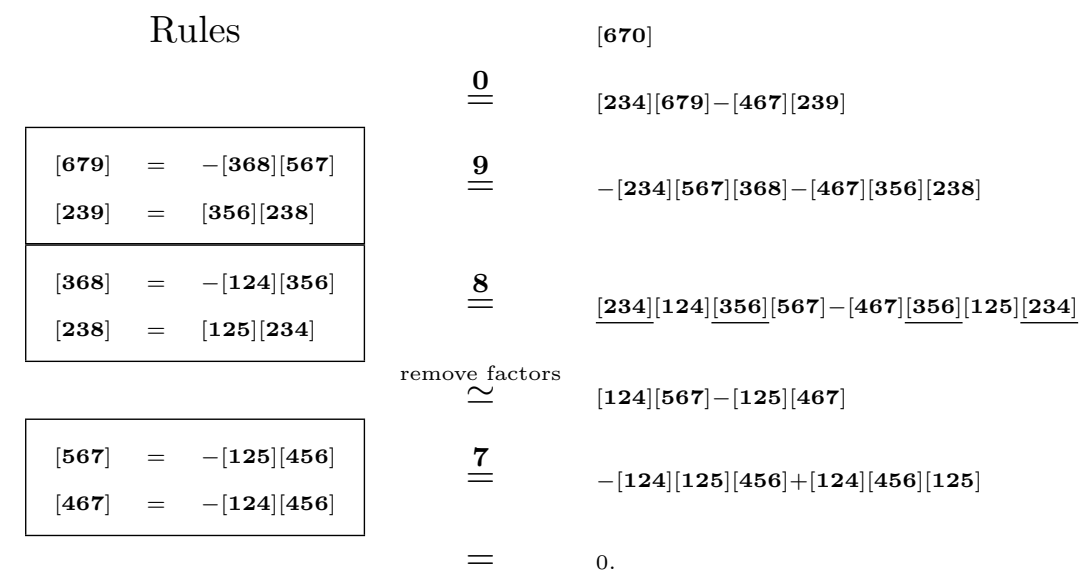

There is no nondegenerate condition.

There is an alternative elimination rule for $\mathbf{0}$. It leads to the second proof of the theorem, which is one step longer because contraction is needed.

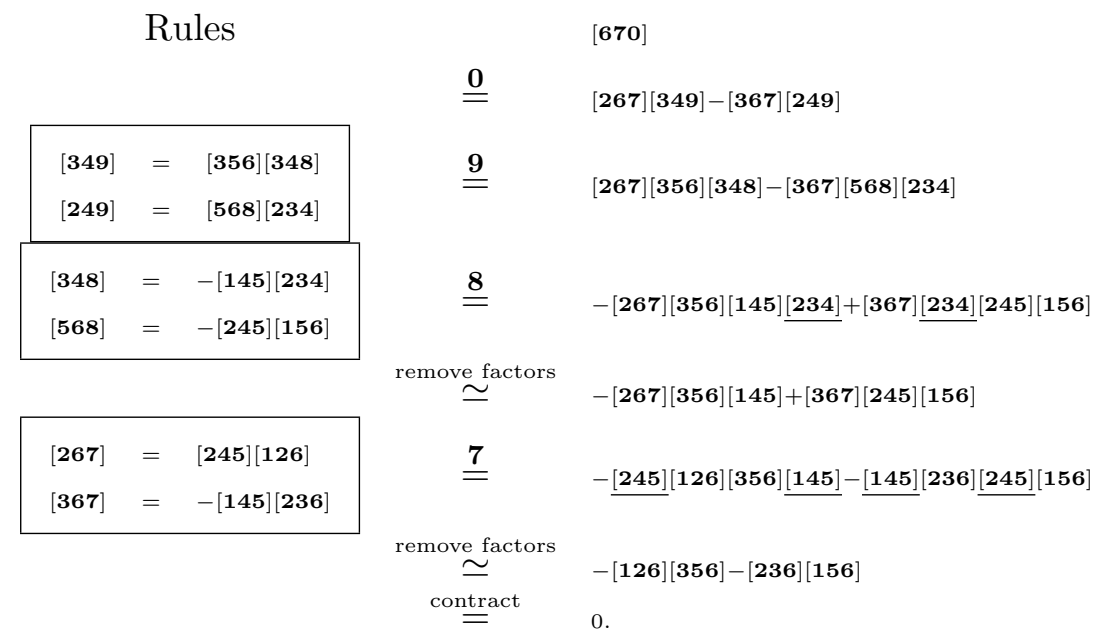

Example 2. 1, 2, 3, 4, 5 are five free points in the projective plane. Point $\mathbf{6}$ is the intersection of lines $\mathbf{3 4}$ and $\mathbf{1 5}$, point $\mathbf{7}$ is the intersection of line $\mathbf{2 6}$ and conic $\mathbf{1 2 3 4 5}$ other than $\mathbf{2}$, points 
$\mathbf{8 , 9}, \mathbf{0}$ are intersections of line pairs 13 and $\mathbf{4 5}, \mathbf{1 4}$ and $\mathbf{3 5}, \mathbf{2 5}$ and $\mathbf{1 7}$, respectively. Then $\mathbf{8 , 9 , 0}$ are collinear.

Proof:

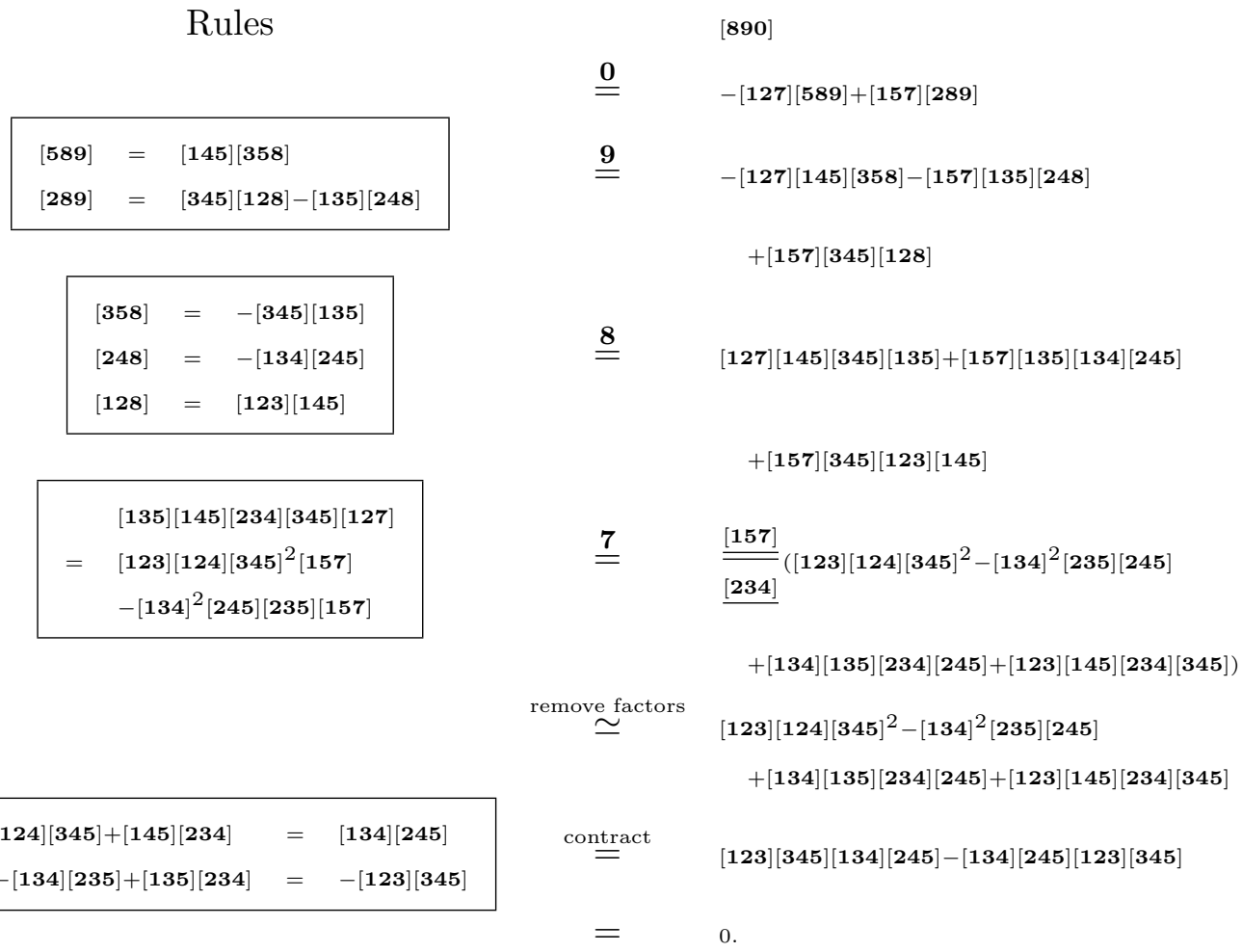

The elimination rules of $\mathbf{7}$ are obtained as follows:

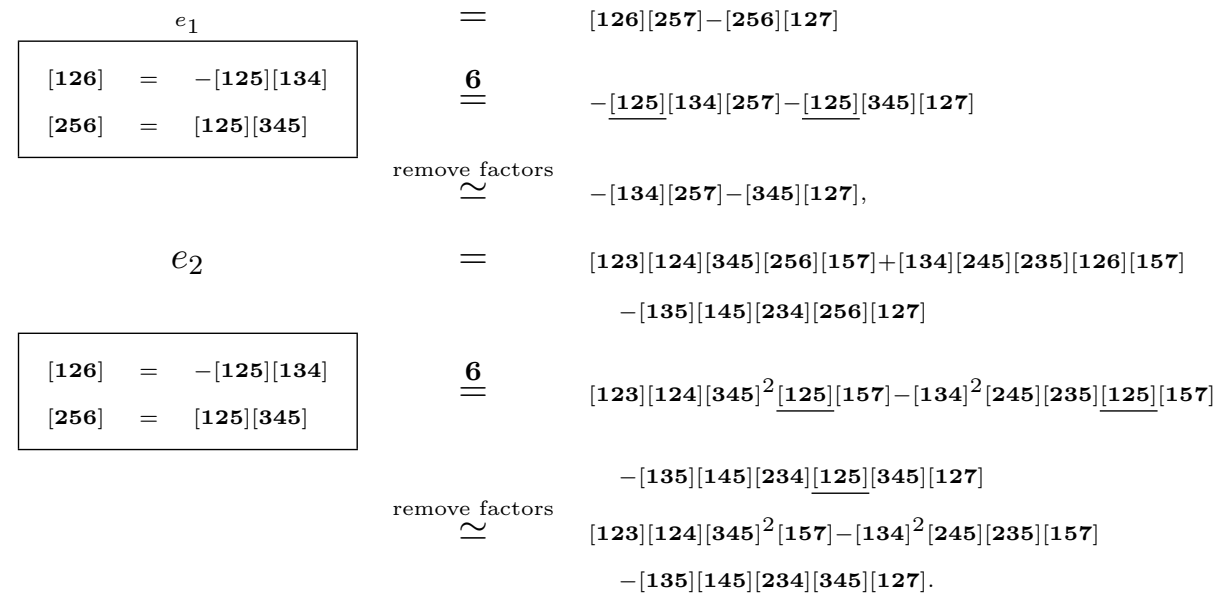

The order of free points when eliminating $\mathbf{7}$ is: $\mathbf{2} \prec \mathbf{1} \prec \mathbf{5} \prec \mathbf{3} \prec \mathbf{4}$.

The nondegenerate condition is $\{[\mathbf{1 2 5}] \neq 0,[\mathbf{2 3 4}] \neq 0\}$.

$\mathbf{0}$ and $\mathbf{9}$ each have an alternative elimination rule. They lead to three other proofs of the theorem. The second proof is much the same as the first one. The third and the fourth 
are much the same, and are each one step longer than the first proof because expansion is needed. The beginning of the third proof is as follows, the later steps are the same with those of the first proof.

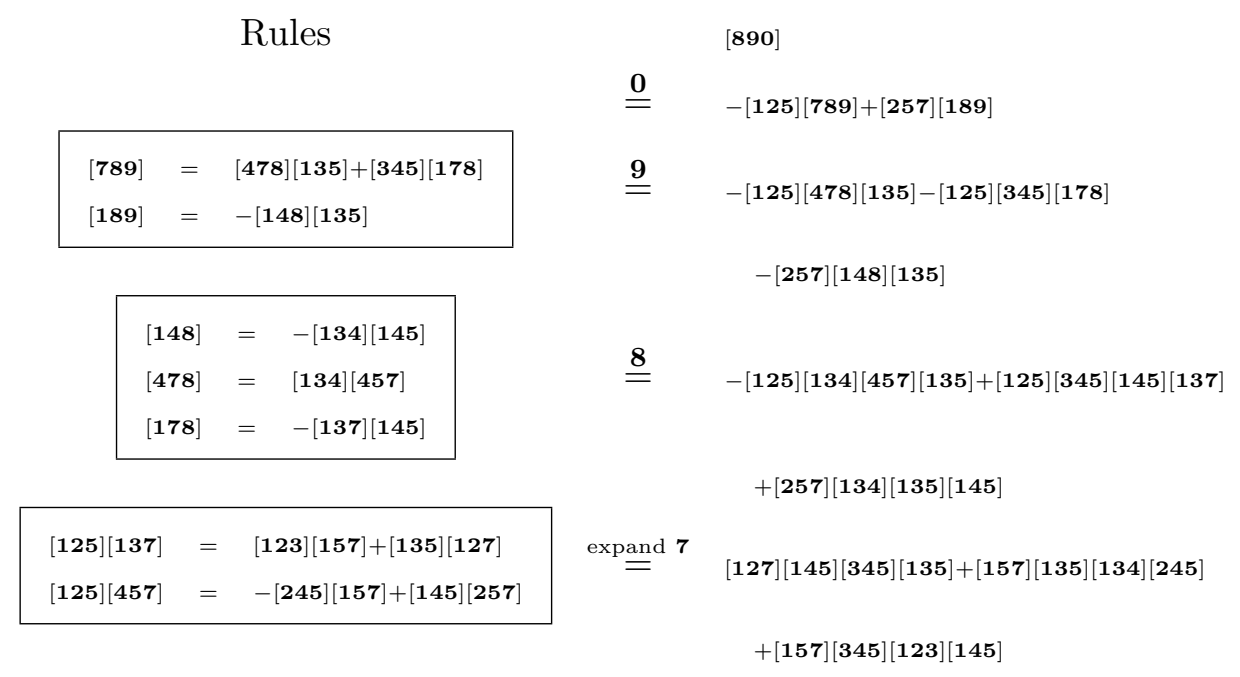

\section{References}

[1] M. Berger. Geometry I, II. Springer, 1987.

[2] B. Buchberger. Application of Gröbner basis in Non-linear Computational Geometry, in: Scientific Software, J. R. Rice (ed.), I.A.M. Vol. in Math. and Its Appl. 444. Springer, New York, 1988.

[3] B. Buchberger. Gröbner Basis-an Algorithmic Method in Polynomial Ideal Theory, in: Multidimensional Systems Theory, N. K. Bose (ed.). 1985.

[4] S.-C. Chou, X.-S. Gao and J.-Z. Zhang. Machine Proofs in Geometry-Automated Production of Readable Proofs for Geometric Theorems. World Scientific, 1994.

[5] H. Crapo and J. Richter-Gebert. Automatic Proving of Geometric Theorems, in: Invariant Methods in Discrete and Computational Geometry, N. White (ed.), 107-139. 1994.

[6] P. Doubilet, G. C. Rota and J. Stein. On the Foundations of Combinatorial Theory IX: Combinatorial Methods in Invariant Theory, Stud. Appl. Math. 57, 185-216. 1974.

[7] S. Fevre and D. Wang. Combining Clifford Algebraic Computing and Term-Rewriting for Geometric Theorem Proving. Fundamenta Informaticae 39 (1-2): 85-104. 1999.

[8] D. Hestenes and R. Ziegler. Projective geometry with Clifford algebra, Acta Appl. Math. 23: 25-63. 1991.

[9] D. Hestenes and G. Sobczyk. Clifford Algebra to Geometric Calculus. D. Reidel, Dordrecht, Boston, 1984.

[10] H.-B. Li and M.-T. Cheng. Proving theorems in elementary geometry with Clifford algebraic method. Chinese Math. Progress 26(4): 357-371. 1997.

[11] H.-B. Li. Vectorial equation-solving for mechanical geometry theorem proving. J. Automated Reasoning. 2000.

[12] H.-B. Li and Y.-H. Wu. Outer Product Factorization in Clifford Algebra, Proc. of ATCM99, pp. 255-264, Guangzhou. 1999. 
[13] B. Mourrain and N. Stolfi. Computational Symbolic geometry, in: Invariant Methods in Discrete and Computational Geometry, N. White (ed.), pp. 107-139. 1994.

[14] J. Richter-Gebert. Mechanical Theorem Proving in Projective Geometry, Annals of Math. and Artificial Intelligence 13, 159-171. 1995.

[15] B. Sturmfels and W. Whitely. On the Synthetic Factorization of Homogeneous Invariants, J. Symbolic Computation 11, 439-454. 1991.

[16] B. Sturmfels. Algorithms in Invariant Theory. Springer, New York, 1993.

[17] D. Wang. Clifford Algebraic Calculus for Geometric Reasoning with Application to Computer Vision. In: Automated Deduction in Geometry, D. Wang (ed.), LNAI 1360, pp. 115-140. Springer, Berlin Heidelberg, 1997.

[18] D. Wang. Clifford Algebraic Computing and Term-rewriting for Geometric Theorem Proving. Proceedings of the 2nd International Theorema Workshop, B. Buchberger and T. Jebelean (eds.), RISC-Linz, Austria. 1998.

[19] W.-T. Wu. On the Decision Problem and the Mechanization of Theorem Proving in Elementary Geometry, Scientia Sinica 21, 159-171. 1978.

[20] W.-T. Wu. Basic Principles of Mechanical Theorem Proving in Elementary Geometries, J. Sys. Sci. \& Math. Sci. 4, 207-235. 1984. 